\title{
Extracellular vesicle associated circRNAs for the diagnosis and prognosis of prostate cancer-a commentary
}

\author{
Elena S. Martens-Uzunova \\ Department of Urology, Erasmus MC Cancer Institute, Erasmus University Medical Center, Rotterdam, The Netherlands \\ Correspondence to: Elena S. Martens-Uzunova. Department of Urology, Erasmus MC, 2040, 3000CA Rotterdam, The Netherlands. \\ Email: e.martens@erasmusmc.nl. \\ Comment on: He YD, Tao W, He T, et al. A urine extracellular vesicle circRNA classifier for detection of high-grade prostate cancer in patients with \\ prostate-specific antigen 2-10 $\mathrm{ng} / \mathrm{mL}$ at initial biopsy. Mol Cancer 2021;20:96.
}

Received: 10 October 2021; Accepted: 29 October 2021; Published: 30 November 2021.

doi: 10.21037/exrna-21-20

View this article at: https://dx.doi.org/10.21037/exrna-21-20

With more than 1.4 million cases in 2020 worldwide, prostate cancer $(\mathrm{PCa})$ is the second most common cancer in men and the fourth most common cancer overall (1). Despite relatively low mortality rates and favorable overall survival, the high incidence of $\mathrm{PCa}$ accounts for a significant number of deaths each year. A main risk factor in $\mathrm{PCa}$ is increased age. This poses a major health concern due to the increasing proportion of elderly man in the general population and the increased risk of over-treatment following the early diagnosis of large proportion of indolent tumors $(2,3)$.

$\mathrm{PCa}$ is usually suspected based on digital rectal examination (DRE) and/or elevated blood levels of Prostate Specific Antigen (PSA, kallikrein-related peptidase 3, KLK). Definitive diagnose is made based on histopathological verification on prostate tissue, obtained by (repeated) transperineal or transrectal biopsies and in some cases during transurethral rejection of the prostate (TURP) to relieve symptoms from benign prostate hyperplasia (BPH), a common pathology in older men $(2,3)$. Subsequent patient stratification into low, intermediate, or high risk cancer is done based on the combination of parameters including PSA levels, and histopathological tumor grade and stage. Unfortunately, PSA has low specificity as a diagnostic biomarker, while transperineal or transrectal biopsy is highly invasive and associated with risk of complications. Furthermore, these procedures do not always give a definitive diagnosis for a considerable proportion of the patients, which undergo subsequent cycles of repetitive biopsies and postponed treatment. On the other hand, many patients that carry indolent low-grade prostate tumors, receive the diagnose cancer, which is often associated with high psychological health burden for them and their families. The successful development of a non-invasive biomarker test that spares invasive biopsy procedures and can detect PCa in body fluids like blood or urine is a currently unmet clinical need. Ideally, such "liquid biopsy" biomarker will detect significant, life-threatening PCa with high specificity and sensitivity, adding value to existing risk calculators in the prevention of overdiagnosis and overtreatment.

In the July issue of Molecular Cancer, He and colleagues (4) address this outstanding challenge by targeting patients at intermediate or high risk of biochemical recurrence of localized or locally-advanced PCa. This patient group is characterized with relatively low PSA levels $(2-10 \mathrm{ng} / \mathrm{mL})$ but biopsy results indicate aggressive tumors with high tumor grade (ISUP prognostic grade group 2 or greater, $\geq$ GG2) (5). He et al. investigate whether a noninvasive liquid biopsy of urine samples could detect high grade PCa before traditional tissue biopsy is taken. The authors take an innovative approach looking to identify prognostic circular RNAs (circRNAs) in the cargo of membrane bound vesicles released in urine from cells of the kidneys, bladder and prostate and referred to as urinary extracellular vesicles $(\mathrm{uEVs})$. The rationale behind this idea is that the molecular cargo of $\mathrm{uEV}$, and in particular proteins and RNA, remain protected from enzymatic and chemical degradation in urine, therefore if $\mathrm{uEV}$ s produced by $\mathrm{PCa}$ cells are present in the urine they may preserve and protect $\mathrm{PCa}$ specific 
molecular biomarkers (6).

The choice for circRNAs as molecular targets of interest is rational, as different types of noncoding RNAs have been shown to play an important role in $\mathrm{PCa}(7,8)$. Among these, the recent discovery of over 70,000 circRNAs in PCa tissue specimens provides a plethora of many potential biomarkers $(9,10)$. Additional advantage is the closed structure of circRNAs - with covalently bound 5'- and 3'-ends, which protects them from the exonucleolytic activity of many ribonucleases. The inclusion of circRNAs in EVs further enhances their stability in body fluids $(10,11)$.

In their study, He et al. follow a robust experimental design starting with a discovery stage, in which they use Next Generation Sequencing (NGS) to identify a set of $18 \mathrm{uEV}$-associated circRNAs, significantly upregulated in PCa patients with high-grade PCa compared to a control group of patients with BPH. To select circRNAs with best discriminative power, the authors measure the expression of these 18 candidates in a large training cohort $(\mathrm{n}=263)$ by digital droplet PCR (ddPCR). In a subsequent machine learning approach tree different statistical models are applied to build the best performing classifier Ccirc, containing 5 most informative circRNAs. The comparison of Ccirc performance with the two most commonly used risk calculators, the Prostate Cancer Prevention Trial Risk Calculator version 2 (PCPR-RC 2.0) (12) for the prediction of low versus high-grade PCa and the European Randomized study of Screening for Prostate Cancer (ERSPC-RC) (13), demonstrates that Ccirc is superior to both risk calculators in the training cohort, as well as in two larger independent validation cohorts $(\mathrm{n}=497$ and $\mathrm{n}=505)$. Integration of Ccirc with either of the risk calculators leads to superior performance in the predicting of high grade PCa ( $\geq$ GG2). Interestingly, Ccirc has better performance compared to other urinary RNA based tests, which relay on DRE to increase the abundance of prostate derived material in urine. A possible reason could be the selection of circRNAs which have higher stability compared to other transcripts present in urine and uEVs. A limitation of the Ccirc is the limited sensitivity as shown by the relatively high percentage of missed cancers $33 \%$ in validation cohort 1 and $25 \%$ in validation cohort 2). A useful future direction would be to evaluate if the integration of Ccirc in the PCPR-RC 2.0 and ERSPC-RC risk calculators would improve detection sensitivity. The expansion of Ccirc with additional circRNAs or other molecular markers is another possible path to explore in this context.

As the authors correctly acknowledge, a major challenge in PCa management remains the ability to discriminate GG2 from GG3 cancers. To address this point they demonstrate that differences in Ccirc values are statistically significant between these two groups, suggesting that this noninvasive assay may have the potential to differentiate individuals with GG2 PCa from those with $\geq$ GG3. Nevertheless, the discriminative power of Ccirc in classifying this two groups remains to be investigated, preferably in specifically designed cohorts. Another important point, which should be the focus of future investigations is whether Ccirc or other similar classifiers would be powerful enough to identify $\mathrm{PCa}$ tumors with invasive cribriform growth pattern. It has been shown that the use of cribriform grade gives better discriminative values for disease-specific and metastasis-free survival than the GG, particularly impacting decision making in men with current GG2 PCa (14-16). The association of cribriform PCa with increased genomic instability and molecular tumor progression (17) supports the existence of specific molecular markers that could be used in "liquid biopsy" assays in the future.

In conclusion, $\mathrm{uEVs}$ and their cargo hold strong potential as clinically applicable source of "liquid biopsy" biomarkers. The work of He et al. discussed above highlights the importance and power of a strong experimental design in biomarker discovery particularly in combination with novel molecular targets and appropriate technical approaches. This paper can therefore be seen as a step forward towards the use of uEVs and their RNA cargo for the diagnosis and prognosis of $\mathrm{PCa}$ in the future clinical practice.

\section{Acknowledgments}

Funding: None.

\section{Footnote}

Provenance and Peer Review: This article was commissioned by the editorial office, $E x R N A$. The article has undergone external peer review.

Conflicts of Interest: The author has completed the ICMJE uniform disclosure form (available at https://dx.doi. org/10.21037/exrna-21-20). The author has no conflicts of interest to declare.

Ethical Statement: The author is accountable for all aspects of the work in ensuring that questions related to the accuracy or integrity of any part of the work are 
appropriately investigated and resolved.

Open Access Statement: This is an Open Access article distributed in accordance with the Creative Commons Attribution-NonCommercial-NoDerivs 4.0 International License (CC BY-NC-ND 4.0), which permits the noncommercial replication and distribution of the article with the strict proviso that no changes or edits are made and the original work is properly cited (including links to both the formal publication through the relevant DOI and the license). See: https://creativecommons.org/licenses/by-nc-nd/4.0/.

\section{References}

1. Bray F, Ferlay J, Soerjomataram I, et al. Global cancer statistics 2018: GLOBOCAN estimates of incidence and mortality worldwide for 36 cancers in 185 countries. CA Cancer J Clin 2018;68:394-424.

2. Mottet N, van den Bergh RCN, Briers E, et al. EAUEANM-ESTRO-ESUR-SIOG Guidelines on Prostate Cancer-2020 Update. Part 1: Screening, Diagnosis, and Local Treatment with Curative Intent. Eur Urol 2021;79:243-62.

3. Cornford P, van den Bergh RCN, Briers E, et al. EAUEANM-ESTRO-ESUR-SIOG Guidelines on Prostate Cancer. Part II-2020 Update: Treatment of Relapsing and Metastatic Prostate Cancer. Eur Urol 2021;79:263-82.

4. He YD, Tao W, He T, et al. A urine extracellular vesicle circRNA classifier for detection of high-grade prostate cancer in patients with prostate-specific antigen 2-10 ng/ $\mathrm{mL}$ at initial biopsy. Mol Cancer 2021;20:96.

5. Pierorazio PM, Walsh PC, Partin AW, et al. Prognostic Gleason grade grouping: data based on the modified Gleason scoring system. BJU Int 2013;111:753-60.

6. Erdbrügger U, Blijdorp CJ, Bijnsdorp IV, et al. Urinary extracellular vesicles: A position paper by the Urine Task Force of the International Society for Extracellular Vesicles. J Extracell Vesicles 2021;10:e12093.

7. Bijnsdorp IV, van Royen ME, Verhaegh GW, et al.

doi: 10.21037/exrna-21-20

Cite this article as: Martens-Uzunova ES. Extracellular Vesicle associated circRNAs for the diagnosis and prognosis of prostate cancer-a commentary. ExRNA 2021;3:9.
The Non-Coding Transcriptome of Prostate Cancer: Implications for Clinical Practice. Mol Diagn Ther 2017;21:385-400.

8. Martens-Uzunova ES, Böttcher R, Croce CM, et al. Long noncoding RNA in prostate, bladder, and kidney cancer. Eur Urol 2014;65:1140-51.

9. Chen S, Huang V, Xu X, et al. Widespread and Functional RNA Circularization in Localized Prostate Cancer. Cell 2019;176:831-843.e22.

10. Chao F, Wang S, Zhang C, et al. The Emerging Role of Circular RNAs in Prostate Cancer: A Systematic Review. Front Cell Dev Biol 2021;9:681163.

11. Shang $Q$, Yang $Z$, Jia R, et al. The novel roles of circRNAs in human cancer. Mol Cancer 2019;18:6.

12. Ankerst DP, Hoefler J, Bock S, et al. Prostate Cancer Prevention Trial risk calculator 2.0 for the prediction of low- vs high-grade prostate cancer. Urology 2014;83:1362-7.

13. Hugosson J, Roobol MJ, Månsson M, et al. A 16yr Follow-up of the European Randomized study of Screening for Prostate Cancer. Eur Urol 2019;76:43-51.

14. van Leenders GJLH, Kweldam CF, Hollemans E, et al. Improved Prostate Cancer Biopsy Grading by Incorporation of Invasive Cribriform and Intraductal Carcinoma in the 2014 Grade Groups. Eur Urol 2020;77:191-8.

15. Kweldam CF, Kümmerlin IP, Nieboer D, et al. Presence of invasive cribriform or intraductal growth at biopsy outperforms percentage grade 4 in predicting outcome of Gleason score 3+4=7 prostate cancer. Mod Pathol 2017;30:1126-32.

16. Downes MR, Xu B, van der Kwast TH. Cribriform architecture prostatic adenocarcinoma in needle biopsies is a strong independent predictor for lymph node metastases in radical prostatectomy. Eur J Cancer 2021;148:432-9.

17. Kweldam CF, van der Kwast T, van Leenders GJ. On cribriform prostate cancer. Transl Androl Urol 2018;7:145-54. 